\title{
Las plantas medicinales en el control de nemátodos gastrointestinales en cabras: potencial de las plantas que crecen en la región de Coquimbo, Chile
}

\author{
MOYA M.A.'; ESCUDERO V.G. ${ }^{*}$ \\ ${ }^{1}$ Grupo de Estudios y Servicios Ambientales del Norte. Calle Ricardo Bitrán N²82, CP: 1700-000, La Serena, \\ Chile., contacto@gesanor.cl; 2 Universidad de La Serena, Facultad de Ciencias, Benavente 980, CP: 1700-000, \\ La Serena, Chile. *Autor para correspondencia: vescudero@userena.cl
}

\begin{abstract}
RESUMO: O objetivo deste estudo foi determinar o potencial de plantas que crecem na região de Coquimbo, no controle de nematódeos gastrintestinais em caprinos. Nós usamos uma abordagem não-experimental para validar a eficácia potencial das plantas medicinais como anti-helmínticos. Esta validação foi realizada com base em uma revisão da extensa literatura consultada a respeito de plantas medicinais. Determinou-se o um potencial de eficácia das plantas, da consistência das informações sobre o uso tradicional, os compostos presentes e farmacologia. Analisamos também os dados dos potenciais efeitos adversos que impedem seu uso em animais. Antecedentes apoiam a um alto potencial da eficácia como anti-helmíntico para se três espécies de plantas que crescem na região de Coquimbo: Allium sativum L., Artemisia absinthium L. e Chenopodium ambrosioides L. Os possíveis efeitos secundários do consumo de plantas medicinais ou seus derivados devem ser avaliadas em testes de campo antes de serem utilizados nas em escala produtiva.
\end{abstract}

Palavras-chave: parasitas gastrointestinais, anti-helmínticos, ervas, cabras.

\begin{abstract}
Medicinal plants in the control of gastrointestinal nematodes in goats: the potential of the plants which grow at the Coquimbo region, Chile. The aim of this study was to determine the potential of the plants which grow at the Coquimbo region in the control of gastrointestinal nematodes in goats. We used a non-experimental approach to evaluate the potential efficacy of medicinal plants as anthelmintics. This research was conducted with exhaustive bibliography referred to the medicinal use of plants in the international literature. We determined the potential of effectiveness of the plants, the consistency of the information about the traditional use, the present compounds and the pharmacology. We also performed an analysis about the potential adverse effects of the plants' application in animals. The collected data support the efficacy of these three species of plants growing at the Coquimbo region as efficient anthelmintic ones: the Allium sativum L., the Artemisia absinthium L. and the Chenopodium ambrosioides $\mathrm{L}$. The possible side effects of the consumption of medicinal plants or their derivatives must be evaluated in field trials before they start being used in a productive scale.
\end{abstract}

Keywords: Gastrointestinal parasites, anthelmintic, medicinal plants, goats.

\section{INTRODUCCIÓN}

La región de Coquimbo presenta diversos climas como el estepárico costero o nuboso, de estepa cálido y templado frío de altura. Es una región de transición ya que se encuentra entre las zonas desértica y templada mediterránea. A lo largo de toda la costa se presenta el clima de tipo estepárico costero o nuboso, el cual su influencia llega hasta el interior hasta 40 kilómetros, por medio de los valles transversales y quebradas. Su mayor característica es la abundante nubosidad; humedad, temperaturas moderadas, con un promedio de precipitaciones de $130 \mathrm{~mm}$ anuales con un período seco de ocho a nueve meses. En la parte interior de la región, por sobre los 800 msnm, se sitúa el clima de estepa cálido que se caracteriza por ausencia de nubosidad y sequedad del aire. Sus temperaturas son mayores que en la costa, las precipitaciones no son tan abundantes y los períodos de sequía son característicos (SIIT, 2014). El $51 \%$ de la superficie de los 3,9 millones de ha de la región se declara 
con alguna utilidad agrícola, regándose solamente el 1,8\%. De los 1,6 millones de ha de la IV Región, el $42 \%$ se considera con aptitud agrícola ganadera, principalmente caprina y ovina. La dotación caprina chilena, según el Censo Nacional Agropecuario de 2007, alcanza a 705.800 cabezas. El $42 \%$ de dichas existencias se concentran en la Región de Coquimbo, con 306.022 cabezas, siendo considerada la región de mayor importancia del país (INDAP, 2009). Según estudios realizados, la superficie capaz de soportar actividad caprina comercial en la región bordea el millón de ha, de las cuales algo menos de la mitad puede soportar una carga de 1 a 4 ha/cabra/año y la otra mitad, 4 a 6 ha/cabra/año (Mujica, 2005). Cabe destacar que una altísima proporción de las cabras chilenas, en especial las de la región de Coquimbo, pertenece a la Agricultura Familiar Campesina, estimativamente sobre el $90 \%$, y es explotada en condiciones de baja eficiencia productiva, con sistemas de explotación tradicionales, de carácter extensivo, aprovechando recursos naturales de bajo costo. (Cofré, 2001; INDAP, 2009). Sin embargo, este sistema de producción genera condiciones propicias para el desarrollo de enfermedades. En este ámbito, destacan las enfermedades parasitarias, las que producen mayores pérdidas económicas que las afecciones bacterianas o virales, debido a su elevado índice de morbilidad, a la merma productiva y en ocasiones a su mortalidad.

Las endoparasitosis y en especial, los parásitos gastrointestinales son de gran importancia en los pequeños rumiantes como las cabras, debido a su particular forma de coger el alimento que tienen estas desde la pradera, lo que hace que puedan ingerir grandes cantidades de larvas infectantes, siendo los animales jóvenes menores de un año los más sensibles a este tipo de parasitosis. Los riesgos de enfermar aumentan con el sobrepastoreo, alta carga animal por hectárea y la mala nutrición; condiciones que son frecuentes de encontrar en una explotación caprina tradicional (Castillo, 2003).

Los efectos del parasitismo gastrointestinal sobre el hospedador son muy diversos, siendo uno de los efectos más frecuentes las úlceras, edema, congestión, necrosis del tracto gastrointestinal, baja en el crecimiento y productividad, y en los casos más severos, la muerte del individuo afectado (Molina, 2004; Gutiérrez, 2007). Esto genera incremento en los costos de producción, debido a la muerte de animales jóvenes y a los gastos inherente a la profilaxis para el tratamiento de animales sintomáticos, afectando la conversión alimenticia y provocando resultados negativos en la producción de leche y carne (Pelegrine, 2006).

Tradicionalmente, el control de los parásitos del tracto digestivo ha estado basado en tratamientos repetitivos con fármacos antihelmínticos (Moreno et al., 2010), pero muchas de las drogas antihelmínticas pueden generar efectos secundarios, como por ejemplo resistencia, toxicidad y reacciones adversas (González et al., 2010).

Un efecto indirecto generado por que el uso indiscriminado de antiparasitarios es la amenaza a la integridad ambiental. Existen antecedentes que confirman que el uso de antiparasitarios provoca cambios en los organismos colonizadores de la materia fecal de animales tratados con estos productos (Iglesias et al., 2005) y también puede generar presencia de residuos tóxicos en alimentos (Alvinerie et al., 1997).

Actualmente se han desarrollado mecanismos alternativos para el control del parasitismo gastrointestinal, como es el uso de plantas medicinales, que mantienen las poblaciones de parásitos bajo los umbrales de pérdidas económicamente aceptables y son menos agresivas con el ambiente. En Chile se conocen y utilizan tradicionalmente cientos de plantas con propiedades medicinales, tanto nativas como introducidas (Tapia, 1995; Avello \& Cisternas, 2010) pero no existen datos en la bibliografía referidos al uso de plantas medicinales, presentes en la región o en el país, para el control de parásitos gastrointestinales en cabras.

En virdud de todos los antecedentes anteriormente señalados, y considerando que la flora nativa y naturalizada que habita en la IV Región de Coquimbo esta compuesta por 1.727 especies, de las cuales 1.478 son nativas de Chile (Marticorena et al., 2001), surgen inmediatamente algunas preguntas al respecto ¿existen plantas nativas o naturalizadas en la región de Coquimbo con propiedades antihelmínticas? ¿Pueden estas plantas ser usadas en cabras? ¿Presentaran efectos secundarios o tóxicos que impidan su uso?

El presente trabajo tiene por objetivo determinar el potencial de las plantas que crecen en la región de Coquimbo en el control de nemátodos gastrointestinales en cabras.

\section{MATERIALES Y MÉTODOS}

Se utilizó un método no experimental para evaluar la eficacia potencial como antihelmínticos de las plantas medicinales, para ello se realizó en revisión exhaustiva de material bibliográfico referido al uso medicinal de las plantas en la bibliografía, tanto el de publicaciones periódicas (a través de las bases de datos disponibles en EBSCO) como en libros; A partir de esta revisión se confeccionó un listado preliminar de los nombres científicos de las plantas citadas en la bibliografía nacional e internacional como vermífugas o antihelmínticas, ya sea de uso en humanos como en animales.

Rev. Bras. PI. Med., Campinas, v.17, n.3, p.480-494, 2015. 
Este listado fue contrastado con los registros de las plantas presentes en la región de Coquimbo (Marticorena et al., 2001), seleccionándose todas las especies que estaban presentes en ambos listados. Esto permitío elaborar un registro preliminar de plantas promisorias, las que fueron agrupadas según su potencial de eficacia presentado por cada especie. Para determinar este potencial de eficacia se utilizó una modificación a la propuesta de Lans et al. (2006), la cual consta de cuatro niveles:

Potencial mínimo: Sólo existe 1 registro de uso popular que apoye el uso buscado. La planta presenta información fitoquímica o farmacológica sobre otros usos.

Potencial aceptable: Existen al menos 2 registros del uso popular de la planta en el tratamiento de enfermedades similares, ya sea en seres humanos o animales.

Potencial medio: Existen más de 2 registros del uso popular de la planta y existe datos fitoquímicos o farmacológicos coherentes con el uso buscado. Registra pruebas in vitro con actividad positiva. La planta puede ejercer una acción fisiológica real

Potencial alto: Todos los antecedentes fitoquímicos y farmacológicos son coherentes con el uso tradicional de la planta. Registra pruebas in vivo con actividad positiva.

De las especies presentes en este registro preliminar promisorio, se seleccionaron aquellas especies vegetales que presentaron al menos un potencial aceptable de eficacia. Para todas las especies seleccionadas se registraron otros antecedentes botánicos (nombre común, familia, estado de conservación en la región y parte de la planta utilizada).

Para poder establecer potenciales más elevados de eficacia antihelmíntica, se consideraron sus propiedades fitoquímicas (principales compuestos), farmacológicas y su uso veterinario. Además se recopilaron datos que podrían limitar su uso en cabras (efectos secundarios, contraindicaciones y toxicidad).

\section{RESULTADOS}

Se encontraron 12 plantas con potencial antihelmintico creciendo en la región de Coquimbo que cumplen con el criterio de potencial aceptable. De ellas, Chenopodium ambrosioides L. Drimys winteri J. R. Forst et G. Forster, Flaveria bidentis (L.) O. Kuntze y Otholobium glandulosum (L.) J. W.Grimes son especies nativas, mientras que las especies Foeniculum vulgare Mill, Ruta chalepensis L. y Tanacetum parthenium (L.) Sch. Bip., son consideradas plantas adventicias en la región, siendo especies cultivadas Allium sativum L.,
Artemisia absinthium L., Juglans regia L., Punica granatum $\mathrm{L}$. y Thymus vulgaris L. (Tabla 1 ).

Las 12 especies selecionadas presentan entre sus componentes flavonoides, lactonas terpénicas o taninos (Tabla 2), sustancias reconocidas por su efecto antihelmíntico. Sin embargo, sólo seis especies, A. sativum, $C$. ambrosoides, $F$. vulgare, J. regia, $P$. granatum y $R$. chalepensis presentan coherencia entre el uso tradicional, datos fitoquímicos, actividad farmacológica (Tabla 2) y ensayos in vitro como antihelmíntico (Tabla 3), alcanzando un potencial de eficiencia medio.

Tres especies presentan un alto potencial de eficiencia como antihelmíntico, con antecedentes de ensayos in vivo para A. sativum, A. absinthium y C. ambrosioides. (Tabla 3).

Se encontraron evidencias de efectos secundarios o de contraindicaciones de gran parte de las plantas seleccionadas por su eficiencia, a excepción J. regia. Los datos referentes a toxicidad indican que especies como $A$. sativum y $P$. granatum no presentan efectos toxicológicos importantes, en cambio en otras especies los estudios toxicológicos han demostrado efectos adversos importantes, como es el caso de $A$. absinthium, $C$. ambrosioides y R. chalepensis (Tabla 4).

\section{DISCUSIÓN}

Son numerosas las plantas que de forma tradicional han sido empleadas como antiparasitarios en humanos y animales en Ámerica (Cáceres \& Machain, 2001; Hall et al., 2002; Terceros, 2007; Satalaya et al., 2009) y en el resto del mundo (Akhtar et al., 2000; Fresquet, 2001; Giday, 2001; Urban et al., 2008). Sin embargo, es notorio el vacío en el conocimiento farmacológico empírico de estas plantas en Chile. La Flora presente en la región de Coquimbo es altamente diversa, sin embargo no se encontraron estudios específicos en relación a las características fitoquímicas o etnobotánicas de las plantas medicinales de esta zona, sólo referencias generales a la condición de "planta medicinal" o datos muy inespecíficos en algunos trabajos.

Una de las plantas con mayor número de estudios asociados es $C$. ambrosoides. Esta especie, originaria de América Central, está ampliamente distribuida y tiene un uso muy extendido a lo largo de toda América, Europa, África y Asia, lo que ha generado una gran cantidad de estudios de sus características y propiedades (Gómez, 2008).

Un elemento importante a considerar al momento de la utilización de una planta medicinal y de sus principios activos son los posibles efectos secundarios que puedan generar, los cuales en algunos casos pueden opacar los efectos terapéuticos. Por ejemplo, en un ensayo realizado en 
TABLA 1. Especies de plantas medicinales usadas tradicionalmente como antihelmínticos presentes en la región de Coquimbo (Potencial de Eficacia Aceptable).

\begin{tabular}{|c|c|c|c|c|c|}
\hline $\begin{array}{l}\text { Nombre } \\
\text { Científico }\end{array}$ & $\begin{array}{l}\text { Nombre } \\
\text { Común }\end{array}$ & Familia & Origen $^{1}$ & $\begin{array}{l}\text { Parte utilizada } \\
\text { como } \\
\text { vermífugo }\end{array}$ & Referencias \\
\hline $\begin{array}{c}\text { Allium sativum } \\
\text { L. }\end{array}$ & Ajo & Alliaceae & Cultivada & Bulbo & $\begin{array}{l}\text { Del Vitto et al. (1998); Fresquet et al. (2001); Giday } \\
\text { (2001); Hall et al. (2002); De la Fuente (2004); Silva et } \\
\text { al. (2004); García (2006); Keller \& Romero (2006); Estrada } \\
\text { et al. (2007); Hermoso (2007); Colombia. Ministerio de } \\
\text { Protección Social (2008); Napoli (2008); Deeba et al. } \\
\text { (2009); } \\
\text { Salazar et al. (2009); Scoles (2006); Scudeller et al. (2009); } \\
\text { Gómez et al. (2011). }\end{array}$ \\
\hline $\begin{array}{c}\text { Artemisia } \\
\text { absinthium L. }\end{array}$ & Ajenjo & Asteraceae & Cultivada & $\begin{array}{c}\text { Hojas, } \\
\text { inflorescencias }\end{array}$ & $\begin{array}{l}\text { Del Vitto et al. (1998); Cáceres \& Machain (2001); Fresquet } \\
\text { et al. (2001); Romo (2001); Scarpa (2002); Quezada } \\
\text { et al. (2005); García (2006); Keller \& Romero (2006); } \\
\text { Bevillacua et al. (2007); Camaqui (2007); Consolini et al. } \\
\text { (2007); Hermoso (2007); Terceros et al. (2007); Colombia. } \\
\text { Ministerio de Protección Social (2008); Bussmann \& Glenn } \\
\text { (2010); Chile. Ministerio de Salud (2010); Grosso (2010); } \\
\text { Guillot (2010); Rodríguez (2010); Muinio (2011). }\end{array}$ \\
\hline $\begin{array}{l}\text { Chenopodium } \\
\text { ambrosioides } \\
\text { L. }\end{array}$ & Paico & Chenopodiaceae & Nativa & Hojas y frutos & $\begin{array}{l}\text { Wilhelm (1992); Del Vitto et al. (1997); Cáceres \& Machain } \\
\text { (2001); Romo (2001); Carrizo et al. (2002); Hernández et } \\
\text { al. (2002); Pérez (2002); Scarpa (2002); Yepes (2004); } \\
\text { Carballo et al. (2005); Quezada et al. (2005); Keller \& } \\
\text { Romero (2006); Scoles (2006); Bevillacua et al. (2007); } \\
\text { Camaqui (2007); Consolini et al. (2007); Estrada et al. } \\
\text { (2007); Gadano et al. (2007); Terceros et al. (2007); Chaves } \\
\text { \& Rodríguez (2008); Gómez (2008); Arias et al. (2009); } \\
\text { Scudeller et al. (2009); Albertasse et al. (2010); Bussmann } \\
\text { et al. (2010); Chile. Ministerio de Salud (2010); Luziatelli et } \\
\text { al. (2010); Martínez \& Barbosa (2010); Rodríguez (2010); } \\
\text { Araujo et al. (2011); Gómez et al. (2011); Huamantupa et } \\
\text { al. (2011); IIAP (2011). }\end{array}$ \\
\hline $\begin{array}{l}\text { Drimys winteri } \\
\text { J. R. Forst et } \\
\text { G. Forster }\end{array}$ & Canelo & Winteraceae & Nativa & Hojas y corteza & $\begin{array}{l}\text { Wilhelm (1992); Montenegro et al. (1994); Massardo \& } \\
\text { Rozzi (1996); Campos (1998); Ibaca (2001); Romo (2001); } \\
\text { Madaleno (2007); Colombia. Ministerio de Protección Social } \\
\text { (2008); Chile. Ministerio de Salud (2010). }\end{array}$ \\
\hline $\begin{array}{l}\text { Flaveria } \\
\text { bidentis (L.) } \\
\text { O. Kuntze }\end{array}$ & Contrayerba & Asteraceae & Nativa & $\begin{array}{l}\text { Hojas, flores y } \\
\text { tallos }\end{array}$ & $\begin{array}{l}\text { Wilhelm (1992); Del Vitto et al. (1997); Pastor \& Zelada } \\
\text { (2006); Dadé et al. (2009); Bussmann et al. (2010); Chile. } \\
\text { Ministerio de Salud (2010). }\end{array}$ \\
\hline
\end{tabular}

\begin{tabular}{|c|c|c|c|c|c|}
\hline $\begin{array}{l}\text { Foeniculum } \\
\text { vulgare Mill }\end{array}$ & Hinojo & Apiaceae & Adventicia & $\begin{array}{l}\text { Semillas, frutos, } \\
\text { hojas y raíz }\end{array}$ & $\begin{array}{l}\text { Mendocilla \& Villar (2001); Chile. Ministerio de Salud (2010); } \\
\text { Kumar et al. (2012). }\end{array}$ \\
\hline $\begin{array}{c}\text { Juglans regia } \\
\text { L. }\end{array}$ & Nogal & Juglandaceae & Cultivada & Hojas & $\begin{array}{l}\text { Del Vitto et al. (1998); García (2006); Hermoso (2007); } \\
\text { Sánchez et al. (2008) Chile. Ministerio de Salud (2010). }\end{array}$ \\
\hline $\begin{array}{l}\text { Otholobium } \\
\text { glandulosum } \\
\text { (L.) J. } \\
\text { W.Grimes }\end{array}$ & Culén & Fabaceae & Nativa & $\begin{array}{l}\text { Toda la planta, } \\
\text { en especial las } \\
\text { hojas }\end{array}$ & $\begin{array}{l}\text { Wilhelm (1992); Montenegro et al. (1994); Erazo et al. } \\
\text { (1997); Hammond et al. (1998); Ibaca (2001); Romo (2001); } \\
\text { Bussmann et al. (2010); Chile. Ministerio de Salud (2010); }\end{array}$ \\
\hline $\begin{array}{c}\text { Punica } \\
\text { granatum L. }\end{array}$ & Granado & Punicaceae & Cultivada & Corteza & $\begin{array}{l}\text { Del Vitto et al. (1998); Lansky et al. (2000); Cáceres \& } \\
\text { Machain (2001); Fresquet et al. (2001); Yepes (2004); } \\
\text { lqbal et al. (2005); Duarte (2006); Keller \& Romero (2006); } \\
\text { Estrada et al. (2007); Godinez \& Volpato (2008); Scudeller } \\
\text { et al. (2009); Chile. Ministerio de Salud (2010); Tomás } \\
\text { (2010); Araujo et al. (2011); Fuenmayor et al. (2011); } \\
\text { Muinio (2011). }\end{array}$ \\
\hline $\begin{array}{c}\text { Ruta } \\
\text { chalepensis L. }\end{array}$ & Ruda & Rutaceae & Adventicia & $\begin{array}{l}\text { Hojas, flores y } \\
\text { tallos }\end{array}$ & $\begin{array}{l}\text { Del Vitto et al. (1998); Giday (2001); Keller \& Romero } \\
\text { (2006); Gonzáles et al. (2007); Napoli (2008); Arias et } \\
\text { al. (2009); Chile. Ministerio de Salud (2010); Rodríguez } \\
\text { (2010); Muinio (2011). }\end{array}$ \\
\hline $\begin{array}{l}\text { Tanacetum } \\
\text { parthenium } \\
\text { (L) Sch.Bip }\end{array}$ & Altamisa & Asteraceae & Adventicia & $\begin{array}{l}\text { Hojas, } \\
\text { inflorescencias } \\
\text { y tallos }\end{array}$ & $\begin{array}{l}\text { Del Vitto et al. (1998); Hammond et al. (1998); Keller \& } \\
\text { Romero (2006); Rateb et al. (2007); Chile. Ministerio de } \\
\text { Salud (2010); Guillot (2010); IIAP (2011). }\end{array}$ \\
\hline $\begin{array}{c}\text { Thymus } \\
\text { vulgaris L. }\end{array}$ & Tomillo & Labiatae & Cultivada & $\begin{array}{l}\text { Hojas, flores y } \\
\text { tallos }\end{array}$ & $\begin{array}{l}\text { Del Vitto et al. (1998); Cáceres \& Machain (2001); Fresquet } \\
\text { et al. (2001); Hall et al. (2002); Quezada et al. (2005); } \\
\text { Duarte (2006); García (2006); Consolini et al. (2007); Chile. } \\
\text { Ministerio de Salud (2010); Rodríguez (2010); Basartea } \\
\text { (2011). }\end{array}$ \\
\hline
\end{tabular}

Rev. Bras. PI. Med., Campinas, v.17, n.3, p.480-494, 2015. 
TABLA 2. Principales compuestos y actividad farmacológica registrada de las especies de plantas medicinales usadas tradicionalmente como antihelmínticos presentes en la región de Coquimbo.

\begin{tabular}{|c|c|c|c|}
\hline $\begin{array}{l}\text { Nombre } \\
\text { Científico }\end{array}$ & Principales compuestos & $\begin{array}{l}\text { Actividad Farmacológica } \\
\text { Antihelmintica Registrada }\end{array}$ & Referencias \\
\hline $\begin{array}{l}\text { Allium sativum } \\
\text { L. }\end{array}$ & $\begin{array}{l}\text { Aliina } \\
\text { Ajoeno } \\
\text { Flavonoides: Apigenina, Quercetina, Miricetina } \\
\text { Saponinas } \\
\text { Aminoácidos }\end{array}$ & $\begin{array}{l}\text { Extracto acuoso: } \\
\text { - Actividad inhibitoria contra nemátodos } \\
\text { gastrointestinales (100mg/ml). } \\
\text { Extracto metanolico: } \\
\text { - Actividad inhibitoria de la motilidad } \\
\text { y sobrevivencia de Haemonchus } \\
\text { contortus } \\
\text { Extracto etanólico: } \\
\text { - Actividad inhibitoria de nemátodos } \\
\text { gastrointestinales ( } 25 \text { y } 50 \mathrm{mg} / \mathrm{ml}) \text {. }\end{array}$ & $\begin{array}{l}\text { Iqbal et al. (2001); Hall } \\
\text { et al. (2002); Colombia. } \\
\text { Ministerio de Protección } \\
\text { Social (2008); Sousa } \\
\text { (2008); Amin et al. (2009); } \\
\text { Reichling et al. (2009). }\end{array}$ \\
\hline $\begin{array}{l}\text { Artemisia. } \\
\text { absinthium L. }\end{array}$ & $\begin{array}{l}\text { Flavonoides } \\
\text { Monoterpenos: a y } \beta \text { Tuyona, Cisocimeno, Canfeno, Pineno, } \\
\text { Felandreno, Acetato de Crisantenilo, Acetato de Sabinilo, Tuyol, Linalol } \\
\text { Lactonas Sesquiterpénicas: Absintina, Artabsina, Anabsina, Anabsinina, } \\
\text { Anabsintina, Artibina, Matricida. } \\
\text { Germacranólidos como Quetopelenólidos. } \\
\text { Flavonoide: Artemisetina, Artemetina, Isoquercitrina, Rutina, Glucósidos } \\
\text { de Patuletina, Isoramnetina, Quercitrina. } \\
\text { Taninos } \\
\text { Ácidos Fenólicos derivados del Ácido Cinámico. } \\
\text { Ácidos Clorogénico, Paracumárico. } \\
\text { Carotenos } \\
\text { Vitamina C. }\end{array}$ & $\begin{array}{l}\text { Extracto eter-etanol: } \\
\text { - Actividad inhibitoria sobre Toxocara } \\
\text { gati }(600 \mathrm{mg} / \mathrm{kg})\end{array}$ & $\begin{array}{l}\text { Colombia. Ministerio de } \\
\text { Protección Social (2008); } \\
\text { Montesdeoca (2009); Yildiz } \\
\text { et al. (2011). }\end{array}$ \\
\hline $\begin{array}{l}\text { Chenopodium } \\
\text { ambrosioides } \\
\text { L. }\end{array}$ & $\begin{array}{l}\text { - } \beta \text {-Myrceno } \\
\text { - } \alpha \text {-Terpineno } \\
\text { - dl-Limoneno } \\
\text { - } \beta \square \text {-Phellandreno } \\
\text { - cis- } \beta \text {-Ocimeno ( } Z \text { ) } \\
\text { - } \psi \text {-Terpineno } \\
\text { - trans- } \beta \text {-Ocimeno } \\
\text { - } p \text {-Cymeno } \\
\text { - } \alpha \text {-Terpinoleno } \\
\text { - } \beta \text {-Caryophylleno } \\
\text { - trans- } p \text {-Mentha-2,8-dien-1-ol } \\
\text { - 1-[2-Methyl-5-(1-methylethenyl) cyclopentyl]-(1 } \alpha, 2 \alpha, 5 \beta) \text {-ethanona } \\
\text { - Citronellyl acetato } \\
\text { - 3,4-Epoxy- } p \text {-menthan-2-ona } \\
\text { - } \gamma \text {-Curcumeno } \\
\text { - Oxido Piperitona } \\
\text { - Cis-Ascaridol } \\
\text { - Trans- } p \text {-Mentha-1(7),8-dien-2-ol } \\
\text { - 3,7-Dimethyl-2,6-octadien-1-ol } \\
\text { - Trans-Ascaridol }\end{array}$ & $\begin{array}{l}\text { Aceite esencial: } \\
\text { - Inhibición de la actividad de larvas } \\
\text { helmintos. } \\
\text { Extracto acuoso: } \\
\text { - Actividad inhibitoria sobre la eclosión } \\
\text { de huevos (ED }{ }_{50}: 0.09 \mathrm{mg} / \mathrm{ml} \text { ) } \\
\text { - Inhibición de la actividad de larvas } \\
\text { helmintos. } \\
\text { Extracto hidroalcoholico: } \\
\text { - Inhibe la actividad de adultos de } H \text {. } \\
\text { contortus }\end{array}$ & $\begin{array}{l}\text { Toso \& Boeris (2001); } \\
\text { Ketzis et al. (2002); Chaves } \\
\text { \& Rodríguez (2008); Singh } \\
\text { et al. (2008); Eguale \& } \\
\text { Giday (2009); Gómez } \\
\text { (2011). }\end{array}$ \\
\hline $\begin{array}{l}\text { Drimys winteri } \\
\text { J. R. Forst et } \\
\text { G. Forster }\end{array}$ & $\begin{array}{l}\text { Taninos } \\
\text { Cíñelo } \\
\text { Ly B Pineno } \\
\text { Manitol } \\
\text { Eugenol } \\
\text { Poligodial } \\
\text { Linalool } \\
\text { B-Cariofileno } \\
\text { L-Humuleno } \\
\text { Farnesol } \\
\text { Flovonoides: Taxifolina y Astilbina } \\
\text { Vitamina C. } \\
\text { Benzocicloheptano } \\
\text { Shyobunona } \\
\text { Azuleno } \\
\text { Naftaleno } \\
\text { Kaureno } \\
\text { - Ciclohexadieno }\end{array}$ & Sin actividad antihelmíntica registrada & $\begin{array}{l}\text { Colombia. Ministerio de } \\
\text { Protección Social (2008); } \\
\text { Becerra et al. (2010). }\end{array}$ \\
\hline
\end{tabular}

Rev. Bras. PI. Med., Campinas, v.17, n.3, p.480-494, 2015. 
TABLA 2. Principales compuestos y actividad farmacológica registrada de las especies de plantas medicinales usadas tradicionalmente como antihelmínticos presentes en la región de Coquimbo.

\begin{tabular}{|c|c|c|c|}
\hline $\begin{array}{l}\text { Flaveria } \\
\text { bidentis (L.) } \\
\text { O. Kuntze }\end{array}$ & $\begin{array}{l}\text { - Eucoantocianidinas } \\
\text { - Esteroides } \\
\text { - Triterpenoides } \\
\text { - -Metil-3-(Metiltio)-Benceno } \\
\text { - 3-Metilbencil Mercaptano } \\
\text { - Flavonoides: 3,7 -Bisulfato de Isoramnetina, 3,4'- Bisulfato de } \\
\text { Quercetina, 3,7,4'- Trisulfato de Quercetina, 3,7,3'- Trisulfato de } \\
\text { Quercetina, 3-Acetil-7,3',4',-Trisulfato de Quercetina, 3,7,3',4', } \\
\text { Tetrasulfato de Quercetina. } \\
\text { - Compuestos Acetilénicos: Tiofenos y Sulfonas no Epoxidadas. }\end{array}$ & Sin actividad antihelmíntica registrada & $\begin{array}{l}\text { Pastor \& Zelada (2006); } \\
\text { Bussmann et al. (2010). }\end{array}$ \\
\hline $\begin{array}{l}\text { Foeniculum } \\
\text { vulgare Mill }\end{array}$ & $\begin{array}{l}\text { - a-Pineno } \\
\text { - Limoneno } \\
\text { - Fenchol } \\
\text { - Anetol } \\
\text { - Anisaldehido } \\
\text { - D-Fenchona, } \\
\text { - 1,8-Cineol } \\
\text { - Cumarinas y Furanocumarinas. } \\
\text { - Flavonoides: Kenferol, Quercetol, Rutósido, Quercitrósido. } \\
\text { - Principios Estrogénicos: Dianetol, Dianisoína. } \\
\text { - B-Sitosterol } \\
\text { - Estigmasterol. } \\
\text { - Felandrene } \\
\text { - Estragol } \\
\text { - Metilchavicol } \\
\text { - Foeniculina } \\
\text { - Safrol } \\
\text { - Ácido Anísico } \\
\text { - Aldehído Anísico } \\
\text { - Cetona Ácianísica } \\
\text { - Enol-P-Propenilfenol. } \\
\text { - Glucorónidos de Flavonoides } \\
\text { - Miristicina } \\
\text { - Feniona } \\
\text { - Alcanfor } \\
\text { - Ácido Clorogénico } \\
\text { - Ácido Caféico. } \\
\text { - Vitaminas A, B y C. }\end{array}$ & $\begin{array}{l}\text { Extracto etanolico y Extracto } \\
\text { acuoso de frutos: } \\
\text { Actividad antihelmíntica probada a } \\
\text { diferentes concentraciones }(25,50 \text {, } \\
100,200 \mathrm{mg} / \mathrm{ml}) \text { sobre Pheritima } \\
\text { posthuma. } \\
\text { Extractos hidroalcohólicos: } \\
\text { Inhibición total de larvas III de } \\
\text { Haemonchus spp. a distintas dosis } \\
(25 \mu \mathrm{l}, 50 \mu \mathrm{l}, 100 \mu \mathrm{l} \text { y } 200 \mu \mathrm{l}) \text {. }\end{array}$ & $\begin{array}{l}\text { Mendocilla \& Villar (2001); } \\
\text { Lamberti et al. (2009); } \\
\text { Kumar et al. (2012) } \\
\text { Sánchez \& Sánchez } \\
\text { (2012). }\end{array}$ \\
\hline $\begin{array}{l}\text { Juglans regia } \\
\text { L. }\end{array}$ & $\begin{array}{l}\text { - Juglona } \\
\text { - Ácidos Hidroxicinnamicos: Ácido 3-caffeoylquinico, Acido } \\
\text { 3-p-coumaroylquinico y } \\
\text { Ácido 4-p-coumaroylquinico. } \\
\text { - Flavonoides: Quercetin } \\
\text { 3-Galactosido, Quercetin 3-Arabinosido, Quercetin 3-Xylosido, } \\
\text { Quercetin 3-Rhamnosido, Quercetin 3-Pentosido y Kaempferol } \\
\text { 3-Pentosido. }\end{array}$ & $\begin{array}{l}\text { Extracto: } \\
\text { - Actividad inhibitoria de motilidad } \\
\text { de larvas maduras de Haemonchus } \\
\text { contortus }\end{array}$ & $\begin{array}{l}\text { Sharma et al. (1971); } \\
\text { Pereira et al. (2007). }\end{array}$ \\
\hline $\begin{array}{l}\text { Otholobium } \\
\text { glandulosum } \\
\text { (L.) J. } \\
\text { W.Grimes }\end{array}$ & $\begin{array}{l}\text { - Fenoles } \\
\text { - Flavonoides } \\
\text { - Taninos } \\
\text { - Cariofileno } \\
\text { - Naftaleno } \\
\text { Ledol } \\
\text { Fenol } \\
\text { Fitol } \\
\text { Eicosano } \\
\text { - Octadecano } \\
\text { - Bakuchiol } \\
\text { - Angelicino } \\
\text { - Psoraleno }\end{array}$ & Sin actividad antihelmíntica registrada & $\begin{array}{l}\text { Niemeyer (1995); Erazo } \\
\text { et al. (1997); Backhouse } \\
\text { et al. (2001); Gutierrez \& } \\
\text { Alva (2006); } \\
\text { Becerra et al. (2010); } \\
\text { Bussmann et al. (2010). }\end{array}$ \\
\hline
\end{tabular}

Rev. Bras. PI. Med., Campinas, v.17, n.3, p.480-494, 2015. 
TABLA 2. Principales compuestos y actividad farmacológica registrada de las especies de plantas medicinales usadas tradicionalmente como antihelmínticos presentes en la región de Coquimbo.

\begin{tabular}{|c|c|c|c|}
\hline $\begin{array}{l}\text { Punica } \\
\text { granatum L. }\end{array}$ & $\begin{array}{l}\text { Polifenoles: Antocianinas, Antocianidinas: Cianidina, Delfinidina, } \\
\text { Pelargonidina. Luteolin, Quercetin y Kaempferol } \\
\text { Ácido Elágico } \\
\text { Ácido Gálico } \\
\text { Ácido Cafeico } \\
\text { - Taninos hidrolizables: Elagitaninos, Punicalaginos, Galotaninos. } \\
\text { - Catequizas } \\
\text { - Esteroides: Estradiol, Estriol, Estrona, Testosterona y Ácido Ursólico. } \\
\text { - Epigalocatequina, } \\
\text { - Hierro } \\
\text { - Aminoácidos. } \\
\text { - Ácido punícico }\end{array}$ & $\begin{array}{l}\text { Extracto alcohólico: } \\
\text { - Inhibe la transformación de huevos a } \\
\text { larvas de Haemonchus contortus } \\
\text { Extracto acuoso: } \\
\text { - Actividad inhibitoria de nemátodos } \\
\text { gastrointestinales }(100 \mathrm{mg} / \mathrm{ml})\end{array}$ & $\begin{array}{l}\text { Iqbal et al. (2005); Amin } \\
\text { et al. (2009); Melgarejo et } \\
\text { al. (2010). }\end{array}$ \\
\hline $\begin{array}{l}\text { Ruta } \\
\text { chalepensis L. }\end{array}$ & $\begin{array}{l}\text { - Alcaloides } \\
\text { - Flavonoides } \\
\text { - Fenoles } \\
\text { - Aminoácidos } \\
\text { - Fucomarinas } \\
\text { - Saponinas } \\
\text { - Limoneno } \\
\text { - Pineno } \\
\text { - Ácido Anímico }\end{array}$ & $\begin{array}{l}\text { Extracto hidroalcohólico: } \\
\text { - Actividad inhibitoria de la motilidad de } \\
\text { Larvas III de Haemonchus spp. }\end{array}$ & $\begin{array}{l}\text { Al -Said et al. (1990); } \\
\text { Gonzáles et al. (2007); } \\
\text { Toso et al. (2007); } \\
\text { Lamberti et al. (2009); } \\
\text { Toribio et al. (2009); } \\
\text { Vazquez et al. (2011) }\end{array}$ \\
\hline $\begin{array}{l}\text { Tanacetum } \\
\text { parthenium } \\
\text { (L.) Sch.Bip }\end{array}$ & $\begin{array}{l}\text { Lactonas Sesquiterpenicas: Partenolido } \\
\text { Flavonoides: Santina, Jaceidina, Centaureidina, Luteolina, Apigenina } \\
\text { Guaianolidos } \\
\text { Alcanfor } \\
\text { Acetato de Crisantenilo } \\
\text { L- Pineno } \\
\text { Limoneno } \\
\text { B-Cariofileno } \\
\text { Eugenol }\end{array}$ & Sin actividad antihelmíntica registrada & $\begin{array}{l}\text { Rateb et al. (2007); } \\
\text { Colombia. Ministerio de } \\
\text { Protección Social (2008). }\end{array}$ \\
\hline $\begin{array}{l}\text { Thymus } \\
\text { vulgaris L. }\end{array}$ & $\begin{array}{l}\text { Timol } \\
\text { Cimene } \\
\text { Carvacrol } \\
\text { Gamma-terpineno } \\
\text { Borneol } \\
\text { Linalol } \\
\text { - Ácido rosmanirico } \\
\text { - Flavonoides: Luteolina, Apigenina, Emidictiol, Cirsilineol, Salvigenina, } \\
\text { Cirsimaritina, Limusina. } \\
\text { - Triterpenos: Ácido Ursolico } \\
\text { - Ácido Salicilico } \\
\text { - Geraniol } \\
\text { - Linalol } \\
\text { - Trans-Tuyanal-Terpineol } \\
\text { - Taninos } \\
\text { - Saponósidos. }\end{array}$ & Sin actividad antihelmíntica registrada & $\begin{array}{l}\text { Hall et al. (2002); Reichling } \\
\text { et al. (2009); Bussmann et } \\
\text { al. (2010). }\end{array}$ \\
\hline
\end{tabular}

el que se aplicó extracto acuoso de $R$. chalepensis a ratones gestantes, se detectaron importantes malformaciones esqueléticas en los embriones (González et al., 2007). Por su parte, el Ascaridol, principal componente del aceite esencial de $C$. ambrosoides es citóxico y genotóxico, incluso en pequeñas dosis pues su efecto es acumulativo (Gadano et al., 2007; Bussman et al., 2011; Gómez et al., 2011). Torres et al (2003) mencionan que su uso en la medicina vernácula no está exento de riesgos, ya que la dosis efectiva está muy cercana a la dosis tóxica, por lo que se deben extremar las precauciones en sus aplicaciones.
Otro elemento a considerar es que la concentración de los principios activos de una planta puede variar dependiendo de factores como la parte de la planta utilizada, la estación del año en que la planta es recolectada o las condiciones del suelo en que crece. A manera de ejemplo mencionaremos las diferencias encontradas en el contenido en Ascaridol en el aceite esencial de $C$. ambrosioides, el cual es muy variable y depende de la zona en que es recolectada. Torres et al. (2003) mencionan que muestras provenientes de San Lorenzo (Corrientes) contenían 10\% de Ascaridol, en La Paz (Córdoba) contenían $20 \%$ y $16 \%$; en Río de Janeiro se dosó

Rev. Bras. PI. Med., Campinas, v.17, n.3, p.480-494, 2015. 
TABLA 3. Especies de plantas medicinales con antecedentes de uso veterinario como antihelmíntico

\begin{tabular}{|c|c|c|c|c|c|}
\hline $\begin{array}{l}\text { Nombre } \\
\text { Científico }\end{array}$ & Enfermedad tratada en animales & Hospedero & Metodología & Lugar & Referencia \\
\hline \multirow[t]{13}{*}{ Allium sativum $\mathrm{L}$. } & Endoparasitos & Rumiantes & Entrevista & Columbia Británica & Lans et al. (2007) \\
\hline & Parasitosis Intestinales (Oxyuriasis) & No especifica & $\begin{array}{c}\text { Revisión } \\
\text { bibliográfica }\end{array}$ & España & Ortiz (2010) \\
\hline & Parásitos intestinales & Gallinas & Entrevista & Uttaranchal, India & Pande et al. (2007) \\
\hline & Antihelmíntico & No especifica & Entrevista & Italia & Guarrera \& Leporatti (2007) \\
\hline & Antihelmíntico & Perros & Entrevista & Italia & Guarrera et al. (2008) \\
\hline & Nemátodos gastrointestinales & Cabras & Ensayo in vitro & Bangladesh, India & Amin et al. (2009) \\
\hline & Parásitos & Ganado & Entrevista & China & Shen et al. (2010) \\
\hline & Parásitos intestinales & No especifica & Entrevista & $\begin{array}{l}\text { Sierras de Córdoba, } \\
\text { Argentina }\end{array}$ & Martínez \& Lujan (2011) \\
\hline & $\begin{array}{l}\text { Nematodiasis por Capillaria sp. y } \\
\text { Spirocamallanus sp. }\end{array}$ & Tilapia y Carpa. & $\begin{array}{c}\text { Revisión } \\
\text { bibliográfica }\end{array}$ & Cuba y México & Prieto et al. (2005) \\
\hline & $\begin{array}{l}\text { Pomphorhynchus laveis y } \\
\text { Acantocephalus anguillae }\end{array}$ & Tilapia & $\begin{array}{c}\text { Revisión } \\
\text { bibliográfica }\end{array}$ & Cuba y México & Prieto et al. (2005) \\
\hline & Parásitos internos & No especifica & Entrevista & Colombia & Gómez et al. (2011) \\
\hline & Helmintos gastrointestinales & Cabras & Ensayo in vitro & Brasil & Sousa (2008) \\
\hline & Haemonchus contortus & Ovejas & Ensayo in vitro & Pakistán & lqbal et al. (2001) \\
\hline \multirow[t]{5}{*}{$\begin{array}{c}\text { Artemisia } \\
\text { absinthium L. }\end{array}$} & $\begin{array}{l}\text { Antihelmíntico, especialmente contra } \\
\text { estróngilos intestinal }\end{array}$ & Ovinos & $\begin{array}{c}\text { Entrevista } \\
\text { y revisión } \\
\text { bibliográfica }\end{array}$ & Uruguay & Grosso (2010) \\
\hline & Contra las tenias como Monezia spp. & Rumiantes & $\begin{array}{c}\text { Entrevista } \\
\text { y revisión } \\
\text { bibliográfica }\end{array}$ & Uruguay & Grosso (2010) \\
\hline & Antiparasitario & Peces & $\begin{array}{l}\text { Revisión } \\
\text { bibliográfica }\end{array}$ & Cuba y México & Prieto et al. (2005) \\
\hline & Lombrices intestinales & Aves de corral & $\begin{array}{c}\text { Entrevista } \\
\text { y revisión } \\
\text { bibliográfica }\end{array}$ & Uruguay & Grosso (2010) \\
\hline & Toxocara cati & Gatos & Ensayo in vivo & Turquía & Yildiz et al. (2011) \\
\hline \multirow[t]{10}{*}{$\begin{array}{l}\text { Chenopodium } \\
\text { ambrosioides } \mathrm{L} \text {. }\end{array}$} & Prevención y control de helmintiasis & Peces & $\begin{array}{c}\text { Revisión } \\
\text { bibliográfica }\end{array}$ & Cuba y México & Prieto et al. (2005) \\
\hline & Antihelmintico & Caballos & Entrevista & $\begin{array}{l}\text { Trinidad y Columbia } \\
\text { Británica (Canadá). }\end{array}$ & Lans et al. (2006) \\
\hline & Huevos de $H$. contortus & Ovejas & Ensayo in vitro & Etiopía & Eguale \& Giday (2009) \\
\hline & Larvas de Haemonchus contortus & Cabras & Ensayo in vitro & USA & Ketzis et al. (2002) \\
\hline & Parasitos internos & Caballos & Entrevista & Chaco Argentina & Scarpa (2000) \\
\hline & Parásitos & Perros & Entrevista & Estelí, Nicaragua & Rodríguez et al. (2005) \\
\hline & Parasitos internos & Aves de corral & Entrevista & $\begin{array}{l}\text { Columbia Británica } \\
\text { (Canadá). }\end{array}$ & Lans \& Turner (2011) \\
\hline & Parásitos internos & Terneros & Entrevista & Caldas, Colombia & Jurado et al. (2007) \\
\hline & Haemonchus contortus & Cabras & Ensayo in vivo & Brasil & Vieira et al. (1999) \\
\hline & Parásitos internos & No especifica & Entrevista & $\begin{array}{l}\text { Costa Atlántica, } \\
\text { Colombia }\end{array}$ & Gómez et al. (2011) \\
\hline $\begin{array}{l}\text { Foeniculum } \\
\text { vulgare Mill }\end{array}$ & $\begin{array}{c}\text { Inhibe la motilidad de larvas III de } \\
\text { Haemonchus spp }\end{array}$ & Ovinos & Ensayo in vitro & Argentina & Lamberti et al. (2009) \\
\hline \multirow[t]{2}{*}{ Juglans regia L. } & Antiparasitario & No especifica & Entrevista & Italia & Guarrera et al. (2008) \\
\hline & $\begin{array}{c}\text { Inhibe la motilidad de larvas maduras } \\
\text { de Haemonchus contortus }\end{array}$ & Cabras & Ensayo in vitro & India & Sharma et al. (1971) \\
\hline \multirow{3}{*}{$\begin{array}{c}\text { Punica granatum } \\
\text { L. }\end{array}$} & Nemátodos gastrointestinales & Cabras & Ensayo in vitro & Bangladesh, India & Amin et al. (2009) \\
\hline & Parásitos intestinales & No especifica & Entrevista & Uttaranchal, India & Pande et al. (2007) \\
\hline & Nemátodos gastrointestinales & Ovejas & $\begin{array}{c}\text { Revisión } \\
\text { Bibliográfica }\end{array}$ & Pakistán & lqbal et al. (2005) \\
\hline \multirow[t]{3}{*}{$\begin{array}{l}\text { Ruta chalepensis } \\
\text { L. }\end{array}$} & Parásitos intestinales & No especifica & Entrevista & $\begin{array}{l}\text { Sierras de Córdoba, } \\
\text { Argentina }\end{array}$ & Martínez \& Lujan (2011) \\
\hline & $\begin{array}{l}\text { Motilidad de Larvas III de } \\
\text { Haemonchus spp. }\end{array}$ & Ovinos & Ensayo in vitro & La Pampa, Argentina & Lamberti et al. (2009) \\
\hline & Parasiticida & Gallinas & Entrevista & Cataluña, España & Bonet \& Valles (2007) \\
\hline
\end{tabular}

Rev. Bras. PI. Med., Campinas, v.17, n.3, p.480-494, 2015. 
TABLA 4. Efectos secundarios, contraindicaciones y toxicidad de las especies de plantas medicinales, con alto potencial de eficiencia como antihelmíntico, presentes en la región de Coquimbo.

\begin{tabular}{|c|c|c|c|c|}
\hline Nombre Científico & Efectos secundarios & $\begin{array}{c}\text { Contraindicaciones y } \\
\text { precauciones }\end{array}$ & Toxicidad & Referencias \\
\hline Allium sativum $\mathrm{L}$. & $\begin{array}{c}\text { Cefaleas, gastritis y diarrea por } \\
\text { sobredosis } \\
\text { Dermatitis de contacto } \\
\text { La ingestión de bulbo de ajos, extracto } \\
\text { de ajo o aceite de ajo podría causar } \\
\text { nausea, vómito, y diarrea, siendo } \\
\text { este último atribuido al contenido de } \\
\text { adenosina como laxante secretomotor. } \\
\text { Exposiciones repetidas a polvos } \\
\text { pueden causar reacciones asmáticas. } \\
\text { Altas concentraciones usadas } \\
\text { externamente pueden causar necrosis } \\
\text { y alergias. }\end{array}$ & $\begin{array}{c}\text { Se debe evitar el uso } \\
\text { concomitante con } \\
\text { anticoagulantes y } \\
\text { antiplaquetarios ya que } \\
\text { puede aumentar el riesgo de } \\
\text { sangrado. }\end{array}$ & $\begin{array}{l}\text { Estudios toxicológicos y } \\
\text { clínicos no han demostrado } \\
\text { efectos adversos } \\
\text { considerables. }\end{array}$ & $\begin{array}{c}\text { Román (2000); } \\
\text { Hall et al. (2002); } \\
\text { Colombia. Ministerio } \\
\text { de Protección Social } \\
\text { (2008). }\end{array}$ \\
\hline Artemisia absinthium L. & $\begin{array}{c}\text { La sobredosis o el uso prolongado } \\
\text { pueden generar síntomas de toxicidad } \\
\text { por Tuyona. }\end{array}$ & $\begin{array}{c}\text { No consumir en caso de } \\
\text { Epilepsia, embarazo e } \\
\text { inflamaciones digestivas } \\
\text { crónicas }\end{array}$ & $\begin{array}{c}\text { DL50 aguda Extracto } \\
\text { fluido: } \\
2499 \mathrm{mg} / \mathrm{Kg} \\
\\
\text { LC50 Extracto acuoso: } \\
93 \mu \mathrm{g} / \mathrm{ml} \\
\text { LC50 Extracto etanólico: } \\
>10000 \mu \mathrm{g} / \mathrm{ml}\end{array}$ & $\begin{array}{l}\text { Del Vitto et al. (1998); } \\
\text { Colombia. Ministerio } \\
\text { de Protección Social } \\
\text { (2008); Bussmann et } \\
\text { al. (2011). }\end{array}$ \\
\hline $\begin{array}{l}\text { Chenopodium } \\
\text { ambrosioides } \mathrm{L} \text {. }\end{array}$ & $\begin{array}{c}\text { El aceite esencial (Ascaridol) es tóxico } \\
\text { al interior. } \\
\text { Nauseas, vómitos, } \\
\text { dolor abdominal } \\
\text { tipo cólico, diarrea } \\
\text { Tóxico por acumulación en pequeñas } \\
\text { dosis }\end{array}$ & $\begin{array}{l}\text { No consumir en caso de } \\
\text { embarazo y lactancia. } \\
\text { Evitar tratamientos } \\
\text { prolongados }\end{array}$ & $\begin{array}{l}\text { LC50 Extracto acuoso: } \\
>1000 \mu \mathrm{g} / \mathrm{ml} \\
\text { LC50 Extracto etanólico: } \\
159 \mu \mathrm{g} / \mathrm{ml} \\
\text { Extracto acuoso: } \\
\quad \text { citotóxico }\end{array}$ & $\begin{array}{l}\text { Pérez (2002); Gadano } \\
\text { et al. (2007); Waizel \& } \\
\text { Waizel (2009); Chile. } \\
\text { Ministerio de Salud } \\
\text { (2010); Bussmann et } \\
\text { al. (2011). }\end{array}$ \\
\hline Foeniculum vulgare Mill & $\begin{array}{l}\text { El anetol y la miristicina, presentes en } \\
\text { el aceite esencial, en dosis mayores } \\
\text { a } 1 \mathrm{ml} \text { pueden provocar efectos } \\
\text { convulsionantes y alucinaciones. }\end{array}$ & $\begin{array}{l}\text { El aceite esencial no debe } \\
\text { emplearse en niños menores } \\
\text { de } 5 \text { años, puede provocar } \\
\text { fotodermatitis y dermatitis por } \\
\text { contacto. }\end{array}$ & $\begin{array}{l}\text { En ratas se observó buena } \\
\text { tolerancia sin ningún efecto } \\
\text { tóxico considerable a dosis } \\
\text { de } 2.000 \mathrm{mg} / \mathrm{kg} .\end{array}$ & $\begin{array}{l}\text { Mendocilla \& Villar } \\
\text { (2001); Colombia. } \\
\text { Ministerio de } \\
\text { Protección Social } \\
\text { (2008); } \\
\text { Sánchez \& Sánchez } \\
\text { (2012). }\end{array}$ \\
\hline Juglans regia L. & Sin información & Sin información & Sin información & \\
\hline Punica granatum $\mathrm{L}$. & $\begin{array}{l}\text { La sobredosis puede provocar vértigo, } \\
\text { disminución de la visión, debilidad, } \\
\text { calambres y temblores convulsivos. }\end{array}$ & $\begin{array}{l}\text { No consumir en caso de } \\
\text { Epilepsia, embarazo y en } \\
\text { menores de } 5 \text { años. }\end{array}$ & Sin información & $\begin{array}{l}\text { Chile. Ministerio de } \\
\text { Salud (2010). }\end{array}$ \\
\hline Ruta chalepensis L. & $\begin{array}{l}\text { En dosis concentradas o sobredosis } \\
\text { pueden producir úlceras estomacales } \\
\text { o intestinales, vómitos, diarreas, } \\
\text { cefalea, temblores, hipotensión y } \\
\text { colapso cardiocirculatorio. } \\
\text { En estado fresco puede producir } \\
\text { fotodermatitis por contacto. }\end{array}$ & $\begin{array}{c}\text { No consumir durante el } \\
\text { embarazo }\end{array}$ & $\begin{array}{l}\text { Extracto acuoso } \\
\quad \text { liofilizado: } \\
\text { Efectos embriotóxicos } \\
\text { en ratones expuestos } \\
\text { durante el período } \\
\text { postimplantacional } \\
\text { (malformaciones } \\
\text { esqueléticas). }\end{array}$ & $\begin{array}{l}\text { Prada et al. (2003); } \\
\text { Gonzáles et al. } \\
\text { (2007); Chile. } \\
\text { Ministerio de Salud } \\
\text { (2010). }\end{array}$ \\
\hline
\end{tabular}

un 58 a $67,5 \%$, y en aceites esenciales de plantas del Caribe y Centro América, un 42 al 90\%. Por lo tanto, sería aconsejable la realización de estudios etnoveterinarios, fitoquímicos y farmacológicos que identifiquen y cuantifiquen con mayor precisión los principios activos y los efectos de las plantas medicinales que crecen en una determinada región o localidad.

La etnoveterinaria no presenta mayor desarrollo en Chile, por lo que no existen antecedentes respecto al uso de las plantas que crecen en el país como antihelmínticos en animales, a excepción de C. ambrosoides que está ampliamente estudiada en cabras y ovejas en otras regiones del mundo. En Etiopía, Eguale \& Giday (2009) evaluaron el efecto de los extractos acuoso e hidroalcohólico de hojas de $C$. ambrosoides sobre huevos y adultos de Haemonchus contortus en ovejas, encontrando que a una concentración menor o igual a $2 \mathrm{mg} /$ $\mathrm{ml}$ se inhibía el eclosión de los huevos pero con un efecto moderado sobre la supervivencia de los parásitos adultos. En los EE.UU., Ketzis et al. (2002), trabajando con aceite esencial de $C$. ambrosioides en cabras infectadas con Haemonchus contortus señalan que no se tienen efectos importantes en tratamientos a corto plazo (0,2 ml/kg por 5-10 días)

Rev. Bras. PI. Med., Campinas, v.17, n.3, p.480-494, 2015. 
pero si tuvieron una eficacia igual al Tiabendazol, sobre la viabilidad de las larvas.

Algunas especies vegetales muestran efectos antihelmínticos importantes in vitro pero no siempre se replican estos resultados en ensayos in vivo y difícilmente igualan a los antihelmínticos sintéticos disponibles (Githiori et al., 2006; Cuellar, 2007; Nery et al., 2009). Por ejemplo, Batatinha et al. (2004), considerando los buenos resultados que mencionaban los estudios in vitro sobre la actividad antihelmíntica del zumo de ajo en caprinos, evaluaron sus efectos sobre nematódos gastrointestinales de caprinos in vivo, encontrando porcentajes de redución del número de huevos y larvas de Strongyloidea inferiores al 95\%, porcentaje logrado en el grupo control. Por su parte, Worku et al. (2009) reportaron que el concentrado de jugo de ajo no presentó un efecto antihelmíntico eficiente en cabras adultas Boer. Resultados igualmente negativos fueron obtenidos por Santos \& Carvalho (2014) al utilizar tintura alcoholica de ajo en un experimento in vivo con ovejas, y por Amin et al. (2008) quienes estudiaron el efecto del extracto acuoso de ajo en cabras. Esta diferencia en los resultados obtenidos también se repite en otras especies medicinales, como es el caso de Artemisia absinthium (Burke et al., 2009). Santos \& Carvalho (2014) señalan que esta variabilidad en los resultados obtenidos por diferentes investigadores para una misma especie vegetal pueden ser atribuídas a factores relacionados ya sea con la propia planta, con la metodología experimental utilizada o con los animales hospederos.

Estas diferencias en la eficacia de las plantas medicinales, en contraste con referencias sobre su uso tradicional o sobre su actividad in vitro, obliga a los futuros investigadores tanto a normalizar los procedimientos con respecto a la metodología de recolección de plantas, preparación de extractos, dilución, dosificación y modo de administración, como también a considerar elementos adicionales al ensayo per-se. En esta misma línea, Athanasiadou et al. (2007) señalan que la variabilidad estacional y ambiental de una planta también puede afectar las propiedades antiparasitarias y hacen notar la importancia de identificar los mecanismos de acción específicos de cada planta y de definir las especies de parásitos a las cuales está destinada. También mencionan que además de las propiedades antiparasitarias, las plantas medicinales pueden tener propiedades anti-nutricionales que pueden afectar el rendimiento del animal y su comportamiento, por lo los futuros estudios requerirán desarrollar un enfoque holístico al momento de evaluar el potencial de las plantas medicinales en el control de parásitos.

A modo de sumario, podemos señalar que los datos recopilados para las plantas que crecen en la región de Coquimbla indican que al menos 6 especies presentan potencial medio como antihelmíntico: $A$. sativum, $C$. ambrosoides, F. vulgare, $J$. regia, $P$. granatum y $R$. chalepensis. De ellas, $A$. sativum, $A$. absinthium y $C$. ambrosoides, presentan antecedentes, fitoquímicos y farmacológicos coherentes con el uso tradicional de la planta y además registran pruebas in vivo con actividad positiva, por lo que pueden ser consideras con un alto potencial como antihelmíntico. Sin embargo, la gran variabilidad en la eficacia reportada para A. sativum, A. absinthium y $C$. ambrosoides hace necesario nuevos estudios para lograr evidencias concretas y repetibles de su actividad antihelmíntica para su uso en ganado caprino, los que deben incluir tanto ensayos in vivo, con animales naturalmente infectados, observaciones de comportamiento en el animal tratado y pruebas de campo, como así también la caracterización y cuantificación de todos los compuestos específicos de la especie vegetal y la caracterización estacional y ambiental de cada planta para la región de estudio.

\section{REFERENCIAS}

AKHTAR, M. et al. Anthelmintic activity of medicinal plants with particular reference to their use in animals in the Indo-Pakistan subcontinent. Small Ruminant Research, v.38, p.99-107, 2000.

ALBERTASSE, $P$. et al. Plantas medicinais e seus usos na comunidade da Barra do Jucu, Vila Velha, ES. Revista Brasileira de Plantas Medicinais, v.12, n.3, p.250-260, 2010.

AL-SAID, M. et al. Studies on Ruta chalepensis, an ancient medicinal herb still used in traditional medicine. Journal of Ethnopharmaeology, v.28, p.305-312, 1990.

ALVINERIE, $M$. et al. Résidus d'ivermectine dans le lait chez la vache laitiere traitée pendant la période de tarissement avec la posologie recommandée par l'Autorisation de Mise sur le Marché. Revue de Médecine Vétérinaire, v.148, n.2, p.115-116. 1997.

AMIN, M. et al. In vitro anthelmintic efficacy of some indigenous medicinal plants against gastrointestinal nematodes of cattle. Journal of the Bangladesh Agricultural University,

v.7, n.1, p.57-61, 2009.

AMIN, M. et al. Effects of garlic, turmeric and betel leaf against gastrointestinal nematodes in cattle. Bangladesh Veterinary Journal v.6, n.1, p.115-119, 2008.

ARAUJO, F. et al. Etnobotanic and etnovet in jacu settlement in Municipality of Pombal-Paraiba - Brazil. Revista Verde, v.6, n.4, p.149 - 156, 2011.

ARIAS, B. et al. Ethnobotanical knowledge in rural communities of Cordoba (Argentina): the importance of cultural and biogeographical factors. Journal of Ethnobiology and Ethnomedicine, v. 5, n.1, p.40, 2009.

ATHANASIADOU, S. et al. Medicinal plants for helminth 
parasite control: facts and fiction. Animal, v.1, n.9, p.1392-1400, 2007.

AVELLO, M. CISTERNAS, I. Fitoterapia, sus orígenes, características y situación en Chile. Revista Médica de Chile, v.138, p.1288-1293, 2010.

BACKHOUSE, C. et al. Active constituents isolated from Psoralea glandulosa L. with antiinflammatory and antipyretic activities. Journal of Ethnopharmacology, v.78, n.1, p. 27-31, 2001.

BASARTEA, J. Plantas silvestres de Pamplona. Ayuntamiento de Pamplona. Pamplona, 2011. 80p.

BATATINHA, M.J.M. et al. Efeitos do suco de alho (Allium sativum Linn.) sobre nematódeos gastrintestinais de caprinos. Ciência Rural, Santa Maria, v.34, n.4, p.12651266, 2004.

BECERRA, J. et al. Actividad de aceites esenciales de canelo, queule, bailahuén y culén frente a hongos fitopatógenos. Boletín Latinoamericano y del Caribe de Plantas Medicinales y Aromáticas, v.9, n.3, p.212215, 2010.

BEVILLACUA, G. et al.Identificação e tecnologia de plantas medicinais da flora de clima temperado. Circular Técnica 61. Ministério da Agricultura, Pecuária e Abastecimento. 2007. 29p.

BONET, B.; VALLĖS, J. Ethnobotany of Montseny biosphere reserve (Catalonia, Iberian Peninsula): Plants used in veterinary medicine. Journal of Ethnopharmacology, v.110, p.130-147, 2007.

BURKE, J. et al. Herbal dewormer fails to control gastrointestinal nematodes in goats. Veterinary Parasitology, v.160, p.168-170, 2009.

BUSSMANN, R.; GLENN, A. Medicinal plants used in Northern Peru for reproductive problems and female health. Journal of Ethnobiology and Ethnomedicine, v.6, n.1, p.30, 2010.

BUSSMANN, R. et al. Antibacterial activity of medicinal plants of Northern Perú - can tradicional applications provide leads for modern science? Indian Journal of Traditional Knowledge, v.9, n.4, p.742-753, 2010.

BUSSMANN, R. et al. Toxicity of medicinal plants used in traditional medicine in Northern Peru. Journal of Ethnopharmacology, v.137, p.121-140, 2011.

CÁCERES, M.; MACHAIN, M.: Manual de uso de hierbas medicinales del Paraguay. Proyecto Paraguay Farmacopea Tradicional, Patrimonio Cultural y Estrategia de Desarrollo. Fundación Celestina Pérez de Almada - Oficina Regional de Ciencias y Tecnología para América Latina y el Caribe UNESCO, 2001. 72p.

CAMAQUI, A. Plantas medicinales. La experiencia de Tinguipaya. 2da. Edición. Proyecto editorial "La Cooperación Italiana en la Región Andina”, 2007. 280p.

CAMPOS, J. Productos forestales no madereros en Chile. Corporación de Investigación Tecnológica, Intec - Chile. Santiago, Chile. Serie Forestal $\mathbf{N}^{\circ} \mathbf{1 0}$. Oficina Regional de la Fao para América Latina y el Caribe. Dirección de Productos Forestales, Fao, Roma. 1998. Disponível em: <http://www.fao.org/docrep/t2368s/t2368s00.htm>. Acesso em: 10 dic. 2012.

CARBALLO, M. et al. Riesgos y beneficios en el consumo de plantas medicinales. Theoria, v.14, n.2, p.95-108, 2005.

CARRIZO, E. et al. Plantas de uso medicinal en la flora de los alrededores de la ciudad de Santiago del Estero
(Argentina). Dominguezia, v.18, n.1, p. 26-35, 2002.

CASTILLO, M. Estudio epidemiológico del parasitismo gastrointestinal en caprinos lecheros en comuna de Purranque, Décima Región de Los lagos, Chile: Periodo otoño-invierno. 2003. 42p. Memoria de Título (Título de Médico Veterinario) - Instituto de Patología Animal, Facultad de Ciencias Veterinarias, Valdivia.

CHAVES, D.; RODRÍGUEZ, R. Evaluación del potencial antihelmíntico de las inflorescencias del apazote, Chenopodium album. Boletín de Parasitología, v.9, n. 3, p.3, 2008.

CHILE. Medicamentos Herbarios Tradicionales. 103 especies vegetales. Red de Protección Social. Ministerio de Salud, Gobierno de Chile, 2010. 231p.

COFRÉ, P. Producción de cabras lecheras. Instituto de Investigaciones Agropecuarias, Chillan, Chile; Boletín INIA N 66; 2001. 134p.

COLOMBIA. Vademécum Colombiano de Plantas Medicinales, Ministerio de Protección Social de Colombia, 2008. 307p. Disponível em: <http:// www.profitocoop.com.ar/articulos/Vademecum $\% 20$ colombiano\%20de\%20plantas $\% 20$ medicinales.pdf>. Acesso em: 05 nov. 2012.

CONSOLINI, A. et al. Estudio observacional del consumo de plantas medicinales en la Provincia de Buenos Aires, Argentina, en el Período diciembre de 2004-noviembre de 2005. Latin American Journal of Pharmacy, v.26, n.6, p.924-36, 2007.

CUELLAR, A. Control no farmacológico de parásitos en ovinos. Nematodos gastroentéricos. Conferencia presentada en el V Congreso de Aleprycs, Mendoza, 22p., 2007. Disponível em: <http://www.produccionanimal.com.ar/ sanidad intoxicaciones metabolicos/ parasitarias/parasitarias_ovinos/02-cuellar.pdf>. Acesso em: 5 de sep. 2013.

DADÉ, M. et al. Total antioxidant capacity and polyphenol content of 21 aqueous extracts obtained from native plants of Traslasierra Valley (Argentina). Boletín Latinoamericano y del Caribe de Plantas Medicinales y Aromáticas, v.8, n.6, p.529-539, 2009.

DEEBA, F. et al. Survey of ethno-veterinary practices used for different ailments in dairy animals in peri-urban areas of Faisalabad (Pakistan). International Journal of Agriculture and Biology, v.11, p.535-541, 2009.

DE LA FUENTE, M. Plantas medicinales para la salud. Editorial Creación, C/ Cartagena, 1428028 - Madrid, 2004. 125p.

DEL VITTO, L. et al. Recursos herbolarios de San Luis (Argentina). Segunda Parte: Plantas exóticas cultivadas, adventicias y/o naturalizadas. Multequina, v.7, p.2948, 1998.

DUARTE, M. Atividade antimicrobiana de plantas medicinais e aromáticas utilizadas no Brasil. MultiCiência: construindo a história dos produtos naturais, v.7, p.1-16, 2006.

EGUALE, T.; GIDAY, M. In vitro anthelmintic activity of three medicinal plants against Haemonchus contortus. International Journal of Green Pharmacy, v.3, p.2934, 2009.

ERAZO, S. et al. Antimicrobial activity of Psoralea glandulosa L. International Journal of

Pharmacognosy and Phytochemical Research, v.35, n.5, p.385-387, 1997.

Rev. Bras. PI. Med., Campinas, v.17, n.3, p.480-494, 2015. 
ESTRADA, E. et al. Ethnobotany in the cumbres de Monterrey National Park, Nuevo León, México. Journal of Ethnobiology and Ethnomedicine, v.3, p.1-8, 2007. FRESQUET, J. et al. Inventario de las plantas medicinales de uso popular en la ciudad de Valencia. Medicina y Ciencias Sociales, n.13, 2001. Disponível em: <http://www.uv.es/medciensoc>. Acesso em: 12 may. 2013.

FUENMAYOR, J. et al. Plantas medicinales presentes en el herbario de la Universidad del Zulia "Omar Zambrano" (HERZU), Revista de la Facultad de Agronomía (LUZ), v.28, s.1, n.396-407, 2011.

GADANO, A. et al. Herbal medicines: cytotoxic effects of Chenopodiaceae species used in Argentinian folk medicine. Pharmaceutical Biology, v.45, n.3, p.217222, 2007.

GARCÍA, C. Control de las helmintosis en ganadería ecológica. Hojas Divulgadoras N²118 HD. Centro de Publicaciones del Ministerio de Agricultura, Pesca y Alimentación. España, 2006. 28p.

GIDAY, M. An ethnobotanical study of medicinal plants used by the Zay people in Ethiopia. CBM: s Skriftserie, v.3, p.81-99, 2001

GITHIORI, J. et al. Use de plants in novel approaches to control of gastrointestinal nematodes in small ruminants. Veterinary Parasitology, v.139, n.4, p.308-320, 2006.

GODINEZ, D; VOLPATO, G. Plantas medicinales que se venden en el mercado El Río, Camagüey, Cuba. Revista Mexicana de Biodiversidad, v.79, p.243-259, 2008.

GÓMEZ, J. Epazote (Chenopodium ambrosioides). Revisión a sus características morfológicas, actividad farmacológica y biogénesis de su principal principio activo, Ascaridol. Boletín Latinoamericano y del Caribe de Plantas Medicinales y Aromática, v.7, n.1, p.3-9, 2008.

GÓMEZ, H. et al. Folk medicine in the northern coast of Colombia: an overview. Journal of Ethnobiology and Ethnomedicine, v.7, n.27, p.1-10, 2011.

GONZALÉS, J. et al. Efecto embriotóxico y teratogénico de Ruta chalepensis L. «ruda», en ratón (Mus musculus). Revista Peruana de Biología, v.13, n.3, p.223 - 225, 2007.

GONZÁLEZ, A. et al. Seguridad de la Ivermectina: toxicidad y reacciones adversas en diversas especies de mamíferos. Revista MVZ Córdoba, v.15, n.2, p.21272135, 2010.

GROSSO, L. El uso popular de las plantas medicinales en Uruguay. La experiencia de los pequeños productores agroecológicos. I Quaderni ZooBioDi n.6. Associazione Italiana di Zootecnia Biologica e Biodinamica. Editado por Lilia Grosso, 2010. 88p.

GUARRERA, P.; LEPORATTI, M. Ethnobotanical remarks on Central and Southern Italy. Journal of Ethnobiology and Ethnomedicine, v.3, n.23, p. 1-11, 2007.

GUARRERA, P. et al. Ethnophytotherapeutical research in the high Molise region (Central-Southern Italy). Journal of Ethnobiology and Ethnomedicine, v.4, n.7, 2008.

GUILLOT, D. La tribu Anthemideae Cass. (Asteraceae) en la flora alóctona de la Península Ibérica e Islas Baleares (Citas bibliográficas y aspectos etnobotánicos e históricos). Monografías de la Revista Bouteloua n.9. Fundación Oroibérico \& Jolube Consultor Editor Ambiental, 2010. 158p. Disponível em: <http://www. floramontiberica.org>. Acesso em: 29 nov. 2012.

GUTIÉRREZ, M. Determinación de parasitismo gastrointestinal mediante el método sodium acetate acetic acid formaldehyde (SAF) en perros de las zonas urbana y rural de la provincia de Ñuble. 2007. 95p. Memoria de Título (Título de Médico Veterinario) - Departamento de Ciencias Pecuarias, Facultad de Medicina Veterinaria, Universidad de Concepción, Concepción.

GUTIERREZ, M.; ALVA, S. Fitoconstituyentes de las hojas de Psoralea glandulosa y efecto del infuso sobre la glicemia en Rattus rattus var. albinus con hiperglicemia experimental. Revista Médica Vallejiana, v.3, n.2, p.85-90, 2006.

HALL, V. et al. Plantas Medicinales de Costa Rica. Volúmen II. Centro Nacional de Información de Medicamentos (CIMED). Facultad de Farmacia. Universidad de Costa Rica, 2002, 135p.

HAMMOND, G. et al. A survey of traditional medicinal plants from the Callejón de Huaylas, Department of Ancash, Perú. Journal of Ethnopharmacology, v.61, p.17-30, 1998.

HERMOSO, C. Los saberes de las mujeres relacionados con las hierbas aromáticas y medicinales y sus aplicaciones en la comarca de Sierra Mágina. Editado por Asociación Para El Desarrollo Rural De Sierra Magina. C/ Posadas, s/n, 23120 Cambil, Jaén, 2007. 193p.

HERNÁNDEZ, J. et al. 23 Especies vegetales medicinales de uso frecuente en la población de Tabay. Revista de la Facultad de Farmacia, v.44, p.51-58, 2002.

HUAMANTUPA, I. et al. Riqueza, uso y origen de plantas medicinales expendidas en los mercados de la ciudad del Cusco. Revista Peruana de Biología, v.18, n.3, p.283 - 291, 2011.

IBACA, R. Monografía de árboles y arbustos chilenos con propiedades medicinales y aromáticas. 2001. 259p. Memoria (Título de Ingeniero Forestal) - Facultad de Ciencias Forestales, Universidad de Concepción, Concepción.

IGLESIAS, L. et al. Impacto ambiental de la Ivermectina eliminada por bovinos tratados en otoño, sobre la coprofauna y la degradación de la materia fecal enpasturas (Tandil, Argentina). Ria, v.34, n.3, p. 83103, 2005.

IIAP - INSTITUTO DE INVESTIGACIONES DE LA AMAZONÍA PERUANA: Base de datos plantas medicinales. Programa de investigación de biodiversidad Amazónica. Proyecto: Prospección y evaluación de sustancias bioactivas y productos naturales. 2011. Disponível em: <http://www.iiap.org. pe/cdpublicaciones2011/documentos/pdf/piba/pu/22. pdf>. Acesso em: 10 dic. 2013.

INDAP - INSTITUTO DE DESARROLLO AGROPECUARIO. Análisis del rubro caprino (leche y carne) en el sector de la agricultura familiar campesina. 2009. Disponível em: <http://www.indap.gob.cl/ ganadero/.../analisis_rubro_caprino_afc.pdf>. Acesso em: 10 may. 2012.

IQBAL, Z. et al. In vitro anthelmintic activity of Allium sativum, Zingiber officinale, Curcurbita mexicana and Ficus religiosa. International Journal of Agriculture and Biology, v.3, n.4, p.454-457, 2001. 
IQBAL, Z. et al. Possible role of ethnoveterinary medicine in poverty reduction in Pakistan: Use of botanical anthelmintics as an example. Journal of Agriculture and Social Sciences, v.1, n.2, p.187-195, 2005.

JURADO, C. et al. Recuperación de los conocimientos tradicionales relacionados con la salud de bovinos a pequeña escala en Villamaría, Caldas, Colombia. Revista Veterinaria y Zootecnia, v.1, n.2, p. 20-29, 2007.

KELLER, H.; ROMERO, H. Plantas medicinales utilizadas por campesinos del área de influencia de la Reserva de Biósfera Yabotí (Misiones, Argentina), Bonplandia, v.15, n.3-4, p.125-141, 2006.

KETZIS, J. et al. Chenopodium ambrosioides and its essential oil as treatments for Haemonchus contortus and mixed adult-nematode infections in goats. Small Ruminantes Research, v.44, p.193-200, 2002.

KUMAR, S. et al. Antihelmintic activity of Cuminum cyminum, Foenicum vulgare and Eugenia caryophyllus against Pheritima posthuma. Indonesian Journal Pharmacology, v.23, n.4, p.225-230, 2012.

LAMBERTI, R. et al. Cribado antihelmíntico de plantas recolectadas en la provincia de La Pampa. Ciencia Veterinaria, v.11, p.26-31, 2009.

LANS, C.; TURNER, N. Organic parasite control for poultry and rabbits in British Columbia, Canada. Journal of Ethnobiology and Ethnomedicine, v.7, n.21, p. 1-9, 2011.

LANS, C. et al. Ethnoveterinary medicines used for horses in Trinidad and in British Columbia, Canada. Journal of Ethnobiology and Ethnomedicine, v.2, n.31, p. 1-20, 2006.

LANS, C. et al. Ethnoveterinary medicines used for ruminants in British Columbia, Canada. Journal of Ethnobiology and Ethnomedicine, v.3, n.11, p. 1-22, 2007.

LANSKY, E. et al. Pharmacological and therapeutic properties of pomegranate. En: Melgarejo, P. et al. Editores. Production, Processing and Marketing of Pomegranate in the Mediterranean Region: Advances in Research and Technology. Options Mediterraneennes, serie A: Seminaries Mediterraneennes. Zaragoza, Spain, v.42, p.231-235, 2000.

LUZIATELLI, G. et al. Asháninka medicinal plants: a case study from the native community of Bajo Quimiriki, Junín, Perú. Journal of Ethnobiology and Ethnomedicine, v.6, n.21, p. 1-23, 2010.

MADALENO, I. Etno-farmacología en Iberoamérica, una alternativa a la globalización de las prácticas de cura. Cuadernos Geográficos, v.41, n.2, p.61-95, 2007.

MARTICORENA, C. et al. Catálogo de la Flora Vascular de la IV Región de Coquimbo. In: Squeo et al. (Eds) Libro Rojo de la Flora Nativa y de los Sitios Prioritarios para su Conservación: Región de Coquimbo. Ediciones Universidad de La Serena, 2001. p. 105-142.

MARTÍNEZ, G.; BARBOSA, G. Natural pharmacopoeia used in traditional Toba medicine for the treatment of parasitosis and skin disorders (Central Chaco, Argentina). Journal of Ethnopharmacology, v.132, p.86-100, 2010.

MARTÍNEZ, G.; LUJÁN, M. Medicinal plants used for traditional veterinary in the Sierras de Córdoba (Argentina): An ethnobotanical comparison with human medicinal uses. Journal of Ethnobiology and Ethnomedicine, v.7, n.23, p. 1-18, 2011.

MASSARDO, F.; ROZZI, R. Valoración de la Biodiversidad: usos medicinales de la flora nativa chilena. Ambiente y Desarrollo, v.12, n.3, p.76 - 81, 1996.

MELGAREJO, P. El granado, su problemática y usos. In: EL GRANADO. I JORNADAS NACIONALES SOBRE EL GRANADO: PRODUCCIÓN, ECONOMÍA, INDUSTRIALIZACIÓN, ALIMENTACIÓN Y SALUD. 2010. Conferencia General. Melgarejo P. et al. Editores, Valencia, 2010. p.7 - 26.

MENDOCILLA, M., VILLAR, M. Monografías de plantas medicinales. In: MANUAL DE FITOTERAPIA. Villar M., Villavicencio O. Editores, Lima, EsSalud, Organización Panamericana de la Salud, 2001. p. 91-345.

MOLINA, B. Medicina etnoveterinaria. Una síntesis bibliográfica. Vétérinaires sans frontières. Lyon, 2004. $34 \mathrm{p}$.

MONTENEGRO, G. et al. Potencialidad de la flora nativa chilena como fuente de productos naturales de uso medicinal. Rojasiana, v.2, n.2, p. 49-66, 1994.

MONTESDEOCA, V. Elaboración y control de calidad de comprimidos fitofarmacéuticos de ajenjo (Arthemisia absinthium L.), romero (Rosmarinus officinalis L.) y manzanilla (Matricaria chamomilla L.) para combatir la menstruación dolorosa. 2009. 142p. Tesis (Título de Bioquímico Farmaceútico). Escuela de Bioquímica y Farmacia, Facultad de Ciencias, Escuela Superior Politécnica de Chimborazo.

MORENO, F. et al. Efecto antihelmíntico in vitro de extractos de plantas sobre larvas infectantes de nematodos gastrointestinales de rumiantes. Archivos de Medicina Veterinaria, v.42, p.155-163, 2010.

MUINIO, W. La Etnobotánica médica del área de transición Pampeano Cuyana. Bonplandia, v.20, n.2, p.353-369, 2011.

MUJICA, F. (Ed.) Razas ovinas y caprinas en el Instituto de Investigaciones Agropecuarias, Osorno, Chile. Instituto de Investigaciones Agropecuarias.Boletín INIA No 127.Osorno, 2005. 88p.

NAPOLI, M. The plants, rituals and spells that 'cured' helminthiasis in Sicily. Journal of Ethnobiology and Ethnomedicine, v.4, n.21, p.1-19, 2008.

NERY, P. et al. Eficácia de plantas para o controle de nematóides gastrintestinais de pequenos ruminantes: revisão de estudos publicados. Revista Brasileira de Plantas Medicinais, v.11, n.3, p.330-338, 2009.

NIEMEYER, H. Biologically active compounds from chilean medicinal plants. In: Amason et al. (Ed.) Phytochemistry of Medicinal Plants. Plenum Press, New York, 1995. p. 137-159.

ORTIZ, N. Plantas de Interés en etnoveterinarina como antiparasitarios e insecticidas en el área de la Dehesa Española. 2010. Disponível em: <http://www. uco.es/zootecniaygestion/img/pictorex/09_10_31_ Plantas_d[1]...pdf>. Acesso em: 5 nov. 2012.

PANDE, P. et al. Ethnoveterinary plants of Uttaranchal -A review. Indian Journal of Traditional Knowledge, v.6, n.3, p.444-458, 2007.

PASTOR, A.; ZELADA, B. Estudio fitoquímico de Flaveria bidentis (L.) Kuntze (Asteraceae). Revista de la Sociedad Química del Perú, v.72, n.1, p.3-11, 2006.

PELEGRINE, A. Efeito anti-helmíntico de taninos

Rev. Bras. PI. Med., Campinas, v.17, n.3, p.480-494, 2015. 
condensados sobre nematódeos gastrintestinais em ovinos. 2006. 164p. Tese do titulo (Doutor em Ciências) - Centro de Energia Nuclear na Agricultura, Comissão de Pós-graduação, Universidade de São Paulo, São Paulo.

PEREIRA, J. et al. Walnut (Juglans regia L.) leaves: Phenolic compounds, antibacterial activity and antioxidant potential of different cultivars. Food and Chemical Toxicology, v.45, p.2287-2295, 2007.

PÉREZ, D. Etnobotánica medicinal y biocidas para malaria en la región Ucayali. Folia Amazónica, v.13, n.1-2, p.87-108, 2002.

PRIETO, A. et al. El empleo de medicina natural en el control de enfermedades de organismos acuáticos y potencialidades de uso en Cuba y México. TIP Revista Especializada en Ciencias Químico-Biológicas, v.8, n.1, p.38-49, 2005.

QUEZADA, F. et al. (Ed.) Biotecnología para el uso sostenible de la biodiversidad. Capacidades locales y mercados potenciales. Corporación Andina de Fomento. Caracas, 2005. 125p.

RATEB, M. et al. Phytochemical and biological investigation of Tanacetum parthenium (L.) cultivated in Egypt. Journal of Medicinal Plants Research, v.1, n.1, p.1826, 2007.

REICHLING, J. et al. Essential oils of aromatic plants with antibacterial, antifungal, antiviral, and cytotoxic properties - an overview. Forsch Komplementmed, v.16, p.79-90, 2009.

RODRÍGUEZ, J. Uso y manejo tradicional de plantas medicinales y mágicas en el valle de Sibundoy, Alto Putumayo, y su relación con procesos locales de construcción ambiental. Revista de la Academia Colombiana de Ciencias Exactas, Físicas y Naturales, v.34, n.132, p.309-326, 2010.

RODRÍGUEZ, O. et al. Plantas utilizadas para el tratamiento de enfermedades en los animales domésticos, Reserva Natural EI Tisey, Estelí. 2005. 152p. Trabajo de Diploma (Título Profesional de Técnico Superior Agropecuario), Estelí.

ROMÁN, C. Determinación de la DL50 y de toxicidad retardada a siete días del extracto de Allium ampeloprasum en ratones. 2000. 36p. Tesis (Grado de Licenciado en Medicina Veterinaria) - Instituto de Farmacología, Facultad de Ciencias Veterinarias, Universidad Austral de Chile, Valdivia.

ROMO, M. Folklore médico de Chiloé. 1.ed. Ediciones del Orfebre, 2001. 47p.

SALAZAR, R. et al. Evaluación de la actividad biológica de productos herbolarios comerciales. Medicina Universitaria, v.11, n.44, p.156-164, 2009.

SÁNCHEZ, I., SÁNCHEZ, A. La diversidad biológica en Cajamarca. Visión étnico-cultural y potencialidades. Editado por Gobierno Regional de Cajamarca, PDRSGIZ, Universidad Nacional de Cajamarca, GRUFIDES. 2012. 205 p.

SÁNCHEZ, A. et al. Uso medicinal de las plantas por los Otomíes del Municipio de Nicolás Flores, Hidalgo, México. Revista Chapingo Serie Horticultura, v.14, n.3, p.271-279, 2008.

SANTOS, F., CARVALHO, N. Alcoholic tincture of garlic (Allium sativum) on gastrointestinal endoparasites of sheep. Ciência Animal Brasileira, v.15, n.1, p. 115-
118, 2014

SATALAYA, J. et al. Actividad antiparasitaria de plantas medicinales de la Amazonía Peruana. Biofarbo, v.17 n.2, p.23-31, 2009.

SCARPA, G. Plants employed in traditional veterinary medicine by the criollos of the northwestern Argentine Chaco. Darwiniana, v.38, n.3-4, p.253-265, 2000.

SCARPA, G. Plantas empleadas contra trastornos digestivos en la medicina tradicional criolla del Chaco Noroccidental. Dominguezia, v.18, n.1, p.36-50, 2002.

SCOLES, R. Sabiduria popular y plantas medicinales: EI ejemplo de la comunidad negra de Itacoã, Acará, Pará. Boletim do Museu Paraense Emílio Goeldi. Ciências Naturais, v.1, n.2, p.79-102, 2006.

SCUDELLER, V. et al. Etnoconhecimento de plantas de uso medicinal nas comunidades São João do Tupé e Central (Reserva de Desenvolvimento Sustentável do Tupé). In: Biotupé: Meio Físico, Diversidade Biológica e Sociocultural do Baixo Rio Negro, Amazônia Central, volumen 2. SANTOS, E.; SCUDELLER, V. (Ed.), UEA Edições, Manaus. 2009. p.185-200.

SHARMA, L. et al. In vitro anthelmintic screening of indigenous medicinal plants against Haemonchus contortus (Rudolphi, 1803) Cobbold, 1898 of sheep and goats. Indian Journal of Animal Research, v.5, p.33-38, 1971.

SHEN S. et al. Ethnoveterinary plant remedies used by Nu people in NW Yunnan of China. Journal of Ethnobiology and Ethnomedicine, v.6, n.24, p.1-10, 2010.

SIIT - SISTEMA INTEGRADO DE INFORMACIÓN TERRITORIAL. Clima y vegetación de la región de Coquimbo. Biblioteca del Congreso Nacional de Chile. Disponível em: <http://siit2.bcn.cl/nuestropais/region4/ clima.htm> Acesso em: 24 mar. 2014

SILVA, F. et al. Plantas Medicinales de la región de Aysen- Chile. Boletín Latinoamericano y del Caribe de Plantas Medicinales y Aromáticas, v.3, n.2, p.3645, 2004

SINGH, H. et al. Chemical composition of essential oil from leaves of Chenopodium ambrosioides from Chandigarh, India. Chemistry of Natural Compounds, v.44, n.3, p.378-379, 2008.

SOUSA, A. Atividade in vitro do extrato etanólico da semente de jerimum (Cucurbita pepo L.) e do suco de alho (Allium sativum L.) em nematóides gastrintestinais de caprinos. 2008. 80p. Dissertação (Programa de Pós-Graduação em Medicina Veterinária de Pequenos Ruminantes e Eqüídeos) - Centro de Saúde e Tecnologia Rural, Pró-Reitoria de PósGraduação e Pesquisa, Universidade Federal de Campina Grande, Patos.

TAPIA, L. Mercado de hierbas medicinales y aceites esenciales. Prediagnóstico y propuesta para la pequeña producción. Centro de Estudios Sociales y Educación. Santiago, Chile, SUR, Centro de Estudios Sociales y Educación (Documento de Trabajo N 150), 1995. 60p.

TERCEROS, P. Plantas medicinales en Bolivia. Estado de arte. Organización de las Naciones Unidas para el Desarrollo Industrial. Subdivisión de Promoción de Inversión y Tecnología. Ministerio de Planificacion 
del Desarrollo Viceministerio de Ciencia y Tecnología Gobierno de Bolivia. 2007. 54p.

TOMÁS, F. Granada y salud: aspectos farmacológicos y terapéuticos de la granada. In: EI Granado. I Jornadas nacionales sobre el granado: Producción, economía, industrialización, alimentación y salud. Melgarejo, P. et al. (Ed.). Publicado por SPE3, S.L., Valencia, 2010. p.205 - 213.

TORIBIO, M. et al. Staphylococcus aureus sensible a extractos metanólicos obtenidos de plantas nativas de la provincia de La Pampa, Argentina. Ciencia Veterinaria, v.11, n.1, p.14-18, 2009.

TORRES, A. et al. Examen del contenido en ascaridol del aceite esencial de Chenopodium ambrosioides $\mathrm{L}$. (Paico). Facena, v.19, p. 27-32, 2003.

TOSO, R.; BOERIS, M. Validación de la actividad antiespasmódica de Sida rhombifolia, Baccharis articulata, Chenopodium ambrosioides y Conyza bonariensis. Ciencia Veterinaria, v.12, n.1, p.20-24, 2001.

TOSO, R. et al. Plantas de la provincia de La Pampa, Argentina, con actividad gastroprotectora y antiespasmódica. Investigación Veterinaria, v.9, n.1, p.145-151, 2007.

URBAN, J. et al. In vitro anthelmintic effects of medicinal plants used in Czech Republic. Pharmaceutical Biology, v.46, n.10-11, p. 808-813, 2008

VÁZQUEZ, B. et al. Uso y conocimiento de plantas medicinales por hombres y mujeres en dos localidades indígenas en Coyomeapan, Puebla, México. Interciencia, v.36, n.7, p.493-499, 2011.

VIEIRA, L. et al. Evaluation of anthelmintic efficacy of plants available in Ceara state, North-east Brazil, for the control of goat gastrointestinal nematodes. Revista de Medicina Veterinaria, v.150, p.447-452, 1999.

WAIZEL, S.; WAIZEL, J. Algunas plantas utilizadas en México para el tratamiento del asma. Revista Anales de Otorrinolaringología Mexicana, v.54, n.4, p.14571, 2009.

WILHELM, E. Botánica Indígena de Chile. Editorial Andrés Bello, Santiago. 1992.136p.

WORKU, M. et al. Efficacy of garlic as an anthelmintic in adult Boer goats. Archives of Biological Science, v.61, n.1, p.135-140, 2009.

YEPES, F. Actuales conocimientos etnobotánicos en Santa Fé de Antioquia. Trabajo presentado en la sesión del Centro de Historia de Santa Fé de Antioquia. 2004. Disponível em: <http:// santafedeantioquia-antioquia.gov.co/apc-aa-fi les/62303466373965613039393633386361/ ACTUALES_CONOCIMIENTOS_ETNOBOTANICOS EN_SANTAFEE_DE_ANTIOQUIA.pdf>. Acesso em: $2 \overline{4}$ nov. 2012.

YILDIZ, K. et al. Antiparasitic efficiency of Artemisia absinthium on Toxocara cati in naturally infected cats. Turkiye Parazitoloji Dernegi, v.35, p.10-4, 2011. 